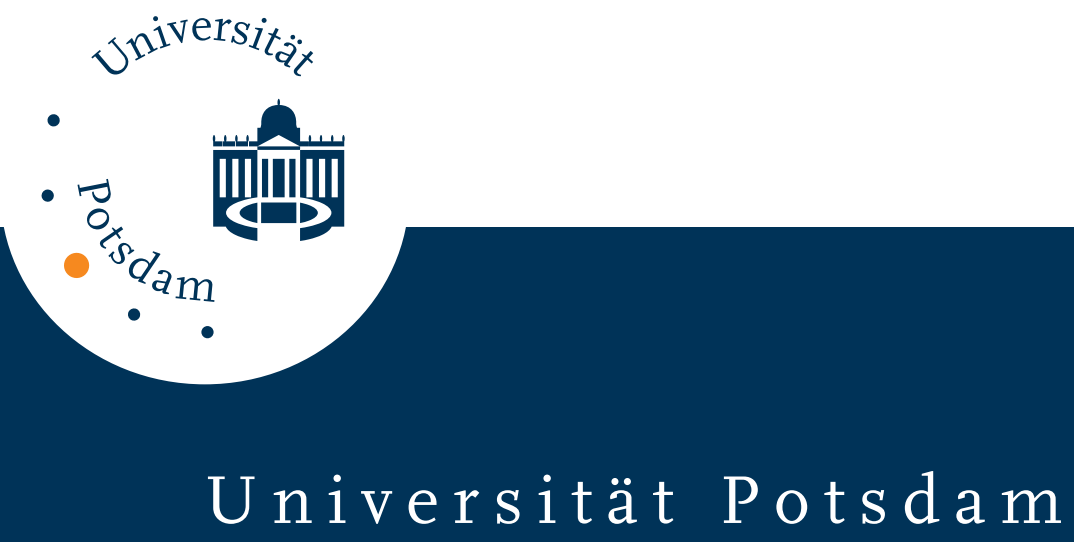

Martin Rolfs, Jochen Laubrock, Reinhold Kliegl

\title{
Shortening and Prolongation of Saccade Latencies Following Microsaccades
}

first published in:

Experimental Brain Research. - ISSN: 0014-4819. - 169 (2006),3 , S. 369-376

Postprint published at the Institutional Repository of the Potsdam University:

In: Postprints der Universität Potsdam

Humanwissenschaftliche Reihe ; 242

http://opus.kobv.de/ubp/volltexte/2011/5701/

http://nbn-resolving.de/urn:nbn:de:kobv:517-opus-57012

Postprints der Universität Potsdam

Humanwissenschaftliche Reihe ; 242 



\section{Shortening and Prolongation of Saccade Latencies Following Microsaccades}

Martin Rolfs, Jochen LaUbrock, Reinhold Kliegl

(UNIVERSITY OF POTSDAM, GERMANY)

Address correspondence to:

Martin Rolfs

Department of Psychology

University of Potsdam

P.O. Box 601553

14415 Potsdam

Germany

E-mail: rolfs@uni-potsdam.de

Phone: +49 (0) 3319772127

Fax: +49(0) 3319772793

Number of pages: 16

Number of figures: 4

Word count (main text, footnotes, figure legends, and references): 5090

Word count (abstract): 194

\section{Acknowledgments}

For helpful conversation and/or comments on earlier drafts of the manuscript, we thank Eike Martin Richter, Ralf Engbert, and two anonymous reviewers. This research was supported by Deutsche Forschungsgemeinschaft (Grants KL955/3 and /6). 


\begin{abstract}
When the eyes fixate at a point in a visual scene, small saccades rapidly shift the image on the retina. The effect of these microsaccades on the latency of subsequent large-scale saccades may be twofold. First, microsaccades are associated with an enhancement of visual perception. Their occurrence during saccade target perception should, thus, decrease saccade latencies. On the other hand, microsaccades likely indicate activity in fixation-related oculomotor neurons. These represent competitors to saccade-related cells in the interplay of gaze holding and shifting. Consequently, an increase in saccade latencies after microsaccades would be expected. Here, we present evidence for both aspects of microsaccadic impact on saccade latency. In a delayed response task, participants made saccades to visible or memorized targets. First, microsaccade occurrence up to $50 \mathrm{~ms}$ before target disappearance correlated with $18 \mathrm{~ms}$ (or $8 \%$ ) faster saccades to memorized targets. Second, if microsaccades occurred shortly (i.e., $<150 \mathrm{~ms}$ ) before a saccade was required, saccadic reaction times in visual and memory trials were increased by about $40 \mathrm{~ms}$ (or 16\%). Hence, microsaccades can have opposite consequences for saccade latencies, pointing at a differential role of these fixational eye movements in preparation of motor programs.
\end{abstract}

Keywords: Fixational eye movements, Memory-guided saccades, Visually-guided saccades 


\section{Introduction}

We scan visual scenes with sequences of saccades (moving gaze) and fixations (holding gaze). During fixations the eyes slowly drift, and, once or twice per second, rapid microsaccades shift the retinal image with amplitudes of less than one degree. Here we test two opposing predictions of how the presence of a microsaccade during a fixation might influence the latency of a subsequent normal saccade.

On the one hand, microsaccades modulate spiking activity in the visual cortex (Bair and O'Keefe 1998; Leopold and Logothetis 1998; Martinez-Conde et al. 2000, 2002; Snodderly et al. 2001; Martinez-Conde et al. 2004, for an overview). Consequently, these fixational eye movements are thought to counteract neuronal adaptation and, thus, to increase visibility (Ditchburn 1980; Gerrits and Vendrik 1974; Martinez-Conde et al. 2000, 2004). Such perceptual enhancement should facilitate the detection of a visual target and, consequently, shorten the latency of a saccadic response towards it.

On the other hand, the physiological mechanisms of microsaccade and saccade generation might interfere. Microsaccades' binocularity (Ditchburn and Ginsborg 1953; Krauskopf et al. 1960; Lord 1951), involuntary occurrence (Ditchburn and Ginsborg 1953; Ratliff and Riggs 1950), and shared kinematic characteristics with large-scale saccades (Zuber et al. 1965) hint at common subcortical mechanisms for the generation of both types of movements (see also discussion). A likely neural correlate of microsaccade generation is the rostral pole of the superior colliculus (SC), a brainstem structure critically involved in the control of saccades and fixations (see Munoz et al. 2000, Scudder et al. 2002, and Sparks 2002, for reviews). The rationale is that saccade amplitudes are coded along the SC rostralcaudal dimension; small amplitudes like those associated with microsaccades being related to activity in the rostral pole (Robinson 1972). Neurons in the rostral pole, however, are associated with active fixation (Munoz and Guitton 1991; Munoz and Wurtz 1993a,b). Consequently, SC activation that causes a microsaccade generates also a longer latency for a subsequent saccade because neurons coding the next saccade target must overcome the activity of these fixation-related neurons in the SC before a saccade may be executed (Munoz and Istvan 1998; Munoz and Wurtz 1993b, 1995b). Thus, if microsaccades result from 
activity in fixation-related neurons in the rostral pole of the SC, they should delay the latency of subsequent saccades.

To our knowledge, neither the proposal that microsaccades originate from fixation-related neurons nor the predictions for the relationship between microsaccades and subsequent saccade latencies have been tested. Here, we show in a single delayed-saccade task that microsaccades are indeed associated with opposite effects in saccadic response latencies. The direction of this effect depends on two factors: (1) when a microsaccade occurs-during the perception of the target or during preparation of a saccade and (2) whether a visually or memory guided saccade is required.

In addition, the present study relates to a recent paper by Supèr et al. (2004). These authors showed that neural activity in V1 increased significantly from 100 ms prior to visually- and memory-guided saccades. Presaccadic visual activity was strongest at saccade target locations. Moreover, Supèr et al. (2004) reported a strong correlation between the strength of presaccadic activity in V1 and latencies of saccades to memorized targets. Based on our earlier research (Engbert and Kliegl 2003; Laubrock et al. 2005; Rolfs et al. 2004, in press), we were interested in how microsaccade statistics relate to Supèr et al.'s results and modeled our delayed-saccade task closely on theirs to facilitate comparison.

\section{Materials and methods}

\section{Participants}

31 students of the University of Potsdam were paid 7 Euro or received study credit for their participation. They were 19 to 40 years old ( $M=24.3$ years), had normal or corrected-to-normal vision and were in good health. This experiment was performed in accordance with the ethical standards laid down in the 1964 Declaration of Helsinki and participants gave their informed consent prior to their inclusion in the study.

\section{Experimental Setup and Eye Movement Recording}

Participants were seated in a silent and darkened room with the head positioned on a chin rest, $50 \mathrm{~cm}$ in front of a computer screen. Stimuli were presented on a 19-inch EYE-Q 650 CRT (1024 by 768 resolution or $40^{\circ}$ by $30^{\circ}$ of visual angle; refresh rate $100 \mathrm{~Hz}$ ). The experiment was controlled by an Apple Power 
Macintosh G4 computer. Eye-position data were recorded and available on-line using an EyeLink-II system (SR Research, Osgoode, ON, Canada) with a sampling rate of $500 \mathrm{~Hz}$ and an instrument spatial resolution better than $0.005^{\circ}$. The experimental software controlling stimulus display and response collection was implemented in Matlab (MathWorks, Natick, Massachusetts, USA), using the Psychophysics (Brainard 1997; Pelli 1997) and EyeLink (Cornelissen et al. 2002) toolboxes.

\section{Procedure}

Participants fixated a point at the center of a computer screen. After $1500 \mathrm{~ms}$ of fixation, a square target appeared at one of three possible positions in the periphery (top: $90^{\circ}$, bottom-left: $210^{\circ}$, or bottom-right: $330^{\circ}$ ). Participants maintained fixation for an additional $1000 \mathrm{~ms}$ until a go signal (fixation point offset) commanded a saccadic response to the target. Response saccades (eye position shift to either of the three target square regions) were detected on-line. If either a response saccade was detected or a response interval of $500 \mathrm{~ms}$ was exceeded, the next trial was started after an inter-trial interval of $500 \mathrm{~ms}$. The sequence of visual stimulation was varied according to three different experimental conditions (see Fig. 1a): (1) The target remained on the screen during the whole fixation period (visual-static condition), (2) it was replaced by a different figure of the same size $280 \mathrm{~ms}$ after target onset (visual-change condition), or (3) it disappeared after $280 \mathrm{~ms}$ of presentation (memory condition). Trials were presented in randomized order, with 102 trials per condition. In addition to stimulus condition (visual-static, visual-change, memory), the factorial design included target position (top, bottom-right, or bottom-left) and target orientation $\left(45^{\circ}\right.$ or $\left.135^{\circ}\right)$.

If gaze position left a fixation square $\left(2^{\circ}\right.$ side length, centered on the fixation spot) during the $2500 \mathrm{~ms}$ fixation period, the trial was aborted. Aborted trials were repeated in random order after the 306 regular trials. Before the first and after every 50 trials the eye tracker was calibrated (standard 9-point grid) and calibration was validated. To start a trial, participants had to fixate a red spot at the center of a random-noise screen (each pixel was set to black or white). Correct fixation was checked, and the stimulus screen appeared if gaze position was detected in the fixation region. Otherwise, a drift correction was carried out and 
the trial was started over. If the eyes were still not detected within the critical area, the calibration was repeated.

\section{Visual Stimuli}

The background of the stimulus screen consisted of a texture of randomly distributed white-on-black line segments of a single orientation. In each trial, a square target (side length $3^{\circ}$ ) was presented at one of three possible locations (north, south-west, or southeast with $4.4^{\circ}$ eccentricity of the square's center from the central red fixation spot of $0.2^{\circ}$ diameter). Target squares set on abruptly and consisted of a random texture of line segments with an orientation orthogonal to the background pattern (see Fig. 1b for an example screen). In two of three conditions, this texture was replaced after $280 \mathrm{~ms}$ by either a backgroundhomogeneous texture (memory trials) or another figure texture (visual-change trials). Line segments were $16 \times 1$ pixels $\left(0.62^{\circ} \times 0.039^{\circ}\right)$ and had an orientation of $135^{\circ}$ or $45^{\circ}$. Both orientations were used for both figure and background, resulting in complementary stimulus pairs. On average, $40 \%$ of the screen were covered by lines.

\section{Data Preparation}

For data analysis, we performed a post-hoc saccade detection based on the algorithm proposed by Engbert and Kliegl (2003). Velocities were computed from subsequent samples in the series of eye positions in the response time window 500 $\mathrm{ms}$ on from the go signal. Saccades were detected in 2D velocity space using thresholds for peak velocity and minimum duration. We used a relative threshold of 6 SDs of the velocity and a minimal duration of $8 \mathrm{~ms}$ (or four data samples).

The first saccade that shifted gaze across one of the three target areas was taken as a response saccade. Saccadic reaction time (SRT) was defined as the latency between go signal and saccade onset.

Subsequently, we used the same algorithm to detect microsaccades (amplitude $<$ $1^{\circ}$ ) in the interval from fixation onset to the response saccade. We considered only binocular microsaccades, that is, microsaccades detected in both eyes with temporal overlap.

Trials including saccades larger than $1^{\circ}$ of visual angle prior to the response saccade were discarded, as were trials with incorrect responses and SRTs shorter than $70 \mathrm{~ms}$. Some trials had to be excluded due to data loss during eye-movement 
recording. Participants contributed 234 to 304 trials to the final data analyses, resulting in a total of 8615 trials (out of 9486 or $90.8 \%$; 2861 visual-static, 2886 visual change, and 2868 memory trials) in which 22529 microsaccades were detected.

\section{Results}

\section{Saccadic Reaction Time}

Mean SRTs were $234 \mathrm{~ms}$ for visual-static, $236 \mathrm{~ms}$ for visual-change, and $238 \mathrm{~ms}$ for memory trials. Differences across conditions were not statistically significant $(F(2,29)=0.830, P=0.49$, ANOVA $)$.

\section{Microsaccade rate}

From former studies we know that the probability of microsaccades evolves in a characteristic signature after stimulus presentation, consisting of an inhibition epoch followed by a temporary enhancement (Engbert and Kliegl 2003; Galfano et al. 2004; Laubrock et al. 2005; Rolfs et al. 2004, in press). Since microsaccade rate is of fundamental importance for the study of microsaccadic influences on SRTs, we first examined this evolution. Fig. 2a shows microsaccade rates across the time course of trials aligned to target onset. Rate evolutions represent averages over participants. Individual rates were computed with a moving time window of $50 \mathrm{~ms}$ centered at time point $t$. When the target remained unchanged on the screen over the entire trial (visual-static), microsaccade rate declined from a relatively stable baseline level of $1.0 \mathrm{~s}^{-1}$ towards a minimum of $0.2 \mathrm{~s}^{-1}$ (inhibition) at about $120 \mathrm{~ms}$. Subsequently, an enhancement of microsaccade rate led to a maximum $\left(2.0 \mathrm{~s}^{-1}\right)$ about $280 \mathrm{~ms}$ after target onset. Finally, rate resettled at a level of $0.7 \mathrm{~s}^{-1}$ (at $380 \mathrm{~ms}$ ), before slightly decreasing, reaching a level of $0.4 \mathrm{~s}^{-1}$ at the time of the go signal. This result replicates the signature of previous research within a new experimental paradigm. During the first $280 \mathrm{~ms}$ visual stimulation was identical and, hence, the rate pattern was not different between visual and memory conditions. However, when a change took place, a second inhibition phase superseded the enhancement phase, leading to a second minimum $\left(0.2 \mathrm{~s}^{-1}\right)$ at about $400 \mathrm{~ms}$. After an additional small enhancement epoch forming a peak of 1.0 $\mathrm{s}^{-1}$ at $550 \mathrm{~ms}$, rates join the slight decrease that was found in the visual-static condition. The second inhibition-enhancement epoch represents a new result in 
agreement with expectations. Note, however, that there was no difference between memory and visual-change conditions in the time course of microsaccade rate, which was thus unaffected by a figure vs. ground interpretation of the change. To study the decrease in microsaccade rate prior to the response saccade, we computed microsaccade onsets relative to saccade onsets. As can be seen in Fig. $2 \mathrm{~b}$, rate drops to a value around $0.1 \mathrm{~s}^{-1}$ just before the saccade.

\section{Microsaccades and Saccadic Response Latencies}

To examine the relation between microsaccades and SRTs, we focus on the difference in SRTs when microsaccades were either present or absent in one of three time windows: First, a pre-target time window $(-100-0 \mathrm{~ms})$ serves as a baseline. Second, a target time window was chosen to test whether microsaccades during target presentation decreased saccade latencies. By setting this time window to $130-230 \mathrm{~ms}$, we ensured a $50 \mathrm{~ms}$ interval between detected microsaccades and target removal in memory trials, allowing for good visual perception of the target after the microsaccade. Third, a pre-saccade time window was set to 900-1000 ms, i.e., just before the go signal. Microsaccades were present (i.e., movement offset within a given interval) in a total of 253 visualstatic, 249 visual-change, and 257 memory trials during the pre-target time window; in respective 126, 114, and 115 trials in the target time window; and in respective 93, 93, and 110 trials in the pre-saccade time window. Mean SRTs are presented in Fig. 3. For each time window ${ }^{1}$, we conducted a $3 \times 2$ repeatedmeasures ANOVA, including the factors condition and microsaccade presence. In the pre-target time window, no significant differences were found; all $F_{\mathrm{S}}<0.9$. In the target time window, again no significant differences were found in the overall tests; all $F s<2.5$ and $P s>0.1$. However, planned orthogonal contrasts revealed an interaction of microsaccade presence with the contrast comparing memory with the average of the visual conditions $(F(1,23)=5.6 ; M S e=810.0 ; P<0.05)$, whereas no interaction was obtained for the contrast comparing SRT in the two visual conditions $(F(1,23)=0.002 ; M S e=1428.7 ; P=0.963)$. For the memory condition, a post-hoc $t$-test for paired samples confirmed that SRTs were shorter if a microsaccade was detected in the target time window ( $240 \mathrm{vs} .222 \mathrm{~ms} ; t(23)=$ $3.2, P<0.005)$. In the pre-saccade time window, there was a main effect of microsaccade presence $(F(1,16)=27.5 ; M S e=1345.4 ; P<0.0001)$, i.e., if

\footnotetext{
${ }^{1}$ Separate ANOVAs were performed because of differences in the number of missing cells.
} 
microsaccades occurred, SRTs were longer than when no microsaccade was observed ( 277 vs. $238 \mathrm{~ms}$ ). There was no interaction of pre-saccadic microsaccade presence with condition $(F(2,15)=0.5 ; M S e=680.8 ; P=0.634)$.

Next, we determined the extension of these effects by moving the chosen time windows in steps of $10 \mathrm{~ms}$ forward and backward in time, respectively. For the resulting time windows, we first computed individual mean SRTs given that either a microsaccade occurred or no microsaccade occurred, and subtracted the latter value from the first. Mean benefits (negative values) and costs (positive values) in saccade latencies $(\triangle S R T)$ embedded in a 95\% confidence interval are displayed in Fig. 4. Based on this analysis, a significant speed-up effect in the memory condition was found continuously in the time windows from $90-190 \mathrm{~ms}$ to 130 $230 \mathrm{~ms}$. Thus, an SRT benefit was observed only if the target was still visible for at least $50 \mathrm{~ms}$ after the microsaccade. The pre-saccadic slowing effect extended back continuously to time windows $790-890 \mathrm{~ms}$ (visual-static), 740-840 ms (visual-change), and 840-940 ms (memory), respectively, after target presentation. This means that SRTs were increased more than $300 \mathrm{~ms}$ after microsaccades, since the minimal possible latency of response saccades was set to $70 \mathrm{~ms}$ in this study. Note, that there were also some significant SRT costs after microsaccades at earlier times (see Fig. 4).

\section{Discussion}

The present study shows for the first time the relationship between microsaccades and latencies of subsequent saccades. First, saccade latencies to memorized target locations were shorter when a microsaccade occurred while the target was transiently presented. This was true despite a delay of roughly one second between target removal and saccade. Second, microsaccades were associated with a pronounced slowing of SRTs if they occurred up to $300 \mathrm{~ms}$ before a saccade had to be executed. Moreover, we obtained the characteristic signature of microsaccade rate evolution in response to display changes in a new experimental paradigm. This is of relevance because, in a recent paper, Supèr et al. (2004) studied pre-saccadic activity in the primary visual cortex of monkeys trained on a delayed saccade task with this paradigm. They showed that V1 activity relating to saccade-target locations increased strongly and continuously from $100 \mathrm{~ms}$ prior to saccade execution. The authors carefully checked for the presence of a higher rate of microsaccades in this time window to rule out fixational movements as a cause 
of this V1 effect and found no modulation by microsaccade rate. Based on our earlier research (Engbert and Kliegl 2003; Rolfs et al. 2004, in press; Laubrock et al. 2005) we expected a substantial microsaccade-rate modulation to show up in a time window different from the ones analyzed by Supèr et al. (2004). Obviously, our results yielded the expected modulation and we think that the same microsaccade rate evolution could also be found in Supèr et al.'s data. ${ }^{2}$

\section{Shortening of saccade latencies following microsaccades}

We provide three speculative explanations for the SRT shortening effect after microsaccades during target presentation in memory trials.

\section{Perceptual-enhancement account Single-cell recordings in monkeys have} shown that microsaccades raise visual responsiveness of neurons in a multitude of brain areas involved in processing of visual information, including the Lateral Geniculate Nucleus (Martinez-Conde et al. 2000, 2002), V1 (Martinez-Conde et al. 2000, 2002; Snodderly et al. 2001; but see Leopold and Logothetis 1998), V2 (Leopold and Logothetis 1998), V4 (Leopold and Logothetis 1998), and MT (Bair and O'Keefe 1998). Since microsaccades relocate the retinal image, these effects have been associated with a refresh of visual information and it was hypothesized that microsaccades might enhance perception of stimuli in the visual periphery (Ditchburn 1980; Gerrits and Vendrik 1974; Martinez-Conde et al. 2000, 2004). If the SRT benefits following microsaccades during target presentation are caused by perceptual enhancements, the question arises why they are restricted to memory-guided saccades. Interestingly, Supèr et al. (2004) also reported a dissociation of visual and memory trials with respect to V1 activity and SRT; they found a strong negative correlation between strength of V1 activity and SRT for memory but not for visual trials. Obviously, a correlation between V1 activity prior to the saccade and the presence of microsaccades during the earlier target presentation in memory but not visual trials would constitute strong evidence for a perceptual-enhancement interpretation restricted to the memory condition. However, given their focus on presaccadic activity in V1, this analysis was not provided by Supèr et al..

\footnotetext{
${ }^{2}$ Criteria for trial rejection applied here partly differed from those used by Supèr et al. (2004). A post-hoc rejection of trials were fixation left an area of $1^{\circ}$ by $1^{\circ}$ (as it was done on-line by Supèr et al.) did not significantly change any of the results reported here.
} 
2. Motor-preparation account An oculomotor area that is highly relevant to the production of memory-guided saccades is the frontal eye fields (FEF). This area receives retinotopically-organized input from the visual cortex (Schall et al. 1995) and contains cells that show tonic discharge activity during the delay periods in memory-saccade tasks. This activity is spatially specific for the target location and available to the target saccade (Friedman et al. 1997; Bruce et al. 2004). In our experiment, conditions were presented in randomized order; the memory condition was certainly the most attention demanding one. Assume that subjects prepared for the disappearance of the target on a fraction of the time and that the occurrence of microsaccades reflects a spillover of enhanced activity in FEF, associated with the preparation of memory-guided saccades during target presentation. In this case, microsaccades are the consequence of task-set related activity. A faster SRT in the memory than the visual conditions would be a consequence of subjects' correct anticipation of a memory trial. And, indeed, in the memory condition SRT was $16 \mathrm{~ms}$ shorter if a microsaccade occurred in the target time window than in the average of the visual conditions; paired $t$-test; $t(23)$ $=1.877, \mathrm{P}=0.037$.

3. Attentional account Finally, there is also an attentional interpretation of shorter SRTs after microsaccades during target presentation in memory trials. In previous work, we linked microsaccades to shifts of covert attention (Engbert and Kliegl 2003; Laubrock et al. 2005; Rolfs et al. 2004, in press). It could be that in those trials where a microsaccade was present this might indicate endogenous shifts of attention to the target incurring an SRT benefit. As the target disappears, there may be more incentive to hold endogenous attention at the remembered target location in the memory condition, whereas in the visual conditions attention may move back to the fixation point. The fact that the microsaccade-related SRT benefit is limited to the memory condition is also compatible with this account. This approach, for the time being, states a pure correlation between microsaccades during target presentation and faster SRTs.

Note that also a combination of the accounts given above might explain the SRT shortening effect. Consider, for instance, that microsaccades during target presentation caused a perceptual enhancement. As a consequence, motorpreparation signals in oculomotor areas might be enhanced. Due to a spillover effect, these might, in turn, result in microsaccades. 


\section{Prolongation of saccade latencies following microsaccades}

Microsaccades share important characteristics with large-scale saccades. First, microsaccades are defined as binocular eye movements with almost identical amplitudes and directions in both eyes (Ditchburn and Ginsborg 1953; Krauskopf et al. 1960; Lord 1951), pointing at a central rather than a peripheral nervous origin. Second, both microsaccades and large-scale saccades fall on the main sequence (Zuber et al. 1965), i.e., the relationship between peak velocity and amplitude in these movements follows a power law. Thus, although the neurophysiological origin of microsaccades is still unknown, it is reasonable to assume a common neural circuitry for the generation of microsaccades and saccades (see also Engbert 2005). Beyond that, microsaccades occur involuntarily (Ditchburn and Ginsborg 1953; Ratliff and Riggs 1950), suggesting that subcortical processes might be most relevant in their production.

A multitude of voluntary and reflexive pathways exists to generate saccades, most of which converge to the $\mathrm{SC}$, the key structure involved in the programming and execution of saccadic eye movements at the subcortical level (see Munoz et al. 2000, Scudder et al. 2002, and Sparks 2002, for reviews). Indeed, there are indications that microsaccades might have a neural correlate in the SC. In detail, the intermediate layers of the SC constitute a retinotopically organized motor map coding for saccades to the contralateral visual field. Here, saccade amplitudes are continuously represented, decreasing from caudal to rostral SC (Robinson 1972). Cells in the rostral pole tonically discharge during fixation and pause or decrease firing during most saccades (Munoz and Guitton 1991; Munoz and Wurtz 1993a). Consequently, they were referred to as fixation neurons (FN) (e.g., Munoz et al. 2000; Munoz and Fecteau 2002; Munoz and Istvan 1998; Munoz and Wurtz 1993a,b, 1995a,b). Saccade-related neurons (SN) are located in the more caudal parts of the SC. SN pause firing during fixations while producing bursts of spikes prior to and during saccades directed to their response field (Sparks et al. 1976; Wurtz and Goldberg 1972; Munoz and Wurtz 1995a). Converging data from microstimulation studies (Gandhi and Keller 1999; Robinson 1972) and single cell recordings (Krauzlis et al. 1997; Munoz and Wurtz 1993a, 1995b) reveal that FN like $\mathrm{SN}$ still possess a movement field, i.e., activity of FN is associated with small 
contraversive saccades. ${ }^{3}$ It is not known whether microsaccades that occur involuntarily during fixation originate in the rostral pole of the $\mathrm{SC}$ as proposed by Gandhi and Keller (1999) and Munoz et al. (2000). Our findings that there is a drop of microsaccade rate prior to saccades and an increase of SRTs in both memory and visual trials when microsaccades occurred around the time of the go signal are consistent with this explanation. As the process of saccade generation requires reciprocal activation of the FN and SN (Munoz and Wurtz 1993b, 1995b; Munoz and Istvan 1998), a decrease in microsaccade rate would be necessary for saccade generation.

The present paper demonstrates on a behavioral basis the differential role of microsaccades in saccade generation. To clarify whether microsaccades are a cause, a correlate, or a consequence of altered saccade dynamics it will be important to determine their neurophysiological correlates. In agreement with our knowledge about the saccadic system, we propose that the SC is a likely candidate at which attentional and perceptual benefits of microsaccades and their inhibiting impact on the generation of large saccades converge.

\section{References}

Bair W, O'Keefe LP (1998) The influence of fixational eye movements on the response of neurons in area MT of the macaque. Visual Neurosci 15:779-786

Brainard DH (1997) The psychophysics toolbox. Spat Vis 10:433-436

Bruce CJ, Friedman HR, Kraus MS, Stanton GB (2004) The primate frontal eye field. In: Chalupa LM , Werner JS (eds), The visual neurosciences, vol 1. MIT, Cambridge, pp 1428-1448

Cornelissen FW, Peters EM, Palmer J (2002) The Eyelink Toolbox: Eye tracking with MATLAB and the Psychophysics Toolbox. Behav Res Meth Ins C 34:613-617

Ditchburn RW (1980) The function of small saccades. Vis Res 20:271-272

Ditchburn RW, Ginsborg BL (1953) Involuntary eye movements during fixation. J Physiol London 119:1-17

Engbert R (2005) Microsaccades: A microcosm for research on oculomotor control, attention, and visual perception. Manuscript submitted

Engbert R, Klieg1 R (2003) Microsaccades uncover the orientation of covert attention. Vis Res 43:1035-1045 Friedman HR, Burman BB, Russo GS, Dias EC, Stanton GB, Shi D, Bruce CJ (1997) Neuronal activity in primate frontal eye field during memory-guided saccades. Soc Neurosci (Abs.) 24:522

Galfano G, Betta E, Turatto M (2004) Inhibition of return in microsaccades. Exp Brain Res 159:400-404 Gandhi NJ, Keller EL (1999) Comparison of saccades perturbed by stimulation of the rostral superior colliculus, the caudal superior colliculus, and the omnipause neuron region. J Neurophysiol 82:3236-3253 Gerrits HJM, Vendrik AJH (1974) The influence of stimulus movements on perception in parafoveal stabilized vision. Vis Res 14:175-180

\footnotetext{
${ }^{3}$ We want to emphasize that the continuum of movement fields along the rostral-caudal dimension extends to saccade amplitudes well below $1^{\circ}$ as observed in the cited studies. Thus, microsaccades fall in the range of amplitudes represented in the very rostral pole of the SC.
} 
Krauskopf J, Cornsweet TN, Riggs LA (1960) Analysis of eye movements during monocular and binocular fixation. J Opt Soc Am 50:572-578

Krauzlis RJ, Basso MA, Wurtz RH (1997) Shared motor error for multiple eye movements. Science

276:1693-1695

Laubrock J, Engbert R, Kliegl R (2005) Microsaccade dynamics during covert attention. Vis Res 45:721-730 Leopold DA, Logothetis NK (1998) Microsaccades differentially modulate neural activity in the striate and extrastriate visual cortex. Exp Brain Res 123:341-345

Lord MP (1951) Measurement of binocular eye movements of subjects in the sitting position. Brit J Ophtal 35:21-30

Martinez-Conde S, Macknik SL, Hubel DH (2000) Microsaccadic eye movements and firing of single cells in the striate cortex of macaque monkeys. Nat Neurosci 3:251-258

Martinez-Conde S, Macknik SL, Hubel DH (2002) The function of bursts of spikes during visual fixation in the awake primate lateral geniculate nucleus and primary visual cortex. Proc Natl Acad Sci USA 99:1392013925

Martinez-Conde S, Macknik SL, Hubel DH (2004) The role of fixational eye movements in visual perception. Nat Rev Neurosci 5:229-240

Munoz DP, Fecteau JH (2002) Vying for dominance: dynamic interactions control visual fixation and saccadic initiation in the superior colliculus. Prog Brain Res 140:3-19

Munoz DP, Guitton D (1991) Control of orienting gaze shifts by the tectoreticulospinal system in the headfree cat II. Sustained discharges during motor preparation and fixation. J Neurophysiol 66:1624-1641 Munoz DP, Istvan PJ (1998) Lateral inhibitory interactions in the intermediate layers of the monkey superior colliculus. J Neurophysiol 79:1193-1209

Munoz DP, Wurtz RH (1993a) Fixation cells in monkey superior colliculus. I. Characteristics of cell discharge. J Neurophysiol 70:559-575

Munoz DP, Wurtz RH (1993b) Fixation cells in monkey superior colliculus. II. Reversible activation and deactivation. J Neurophysiol 70:576-589

Munoz DP, Wurtz RH (1995a) Saccade-related activity in monkey superior colliculus. I. Characteristics of burst and buildup cells. J Neurophysiol 73:2313-2333

Munoz DP, Wurtz RH (1995b) Saccade related activity in monkey superior colliculus. II. Spread of activity during saccades. J Neurophysiol 73:2334-2348

Munoz DP, Dorris MC, Paré M, Everling S (2000) On your mark get set: brainstem circuitry underlying saccadic initiation. Can J Physiol Pharm 78:934-944

Pelli DG (1997) The VideoToolbox software for visual psychophysics: Transforming numbers into movies. Spat Vis 10:437-442

Ratliff F, Riggs LA (1950) Involuntary motions of the eye during monocular fixation. J Exp Psychol London 40:687-701

Robinson DA (1972) Eye movements evoked by collicular stimulation in the alert monkey. Vis Res 12:17951808

Rolfs M, Engbert R, Kliegl R (2004) Microsaccade orientation supports attentional enhancement opposite a peripheral cue. Psychol Sci 15:705-707

Rolfs M, Engbert R, Kliegl R (in press) Crossmodal coupling of oculomotor control and spatial attention in vision and audition. Exp Brain Res

Schall JD, Morel A, King DJ, Bullier J (1995) Topography of visual cortex connections with frontal eye field in macaque: Convergence and segregation of processing streams. J Neurosci 15:4464-4487

Scudder CA, Kaneko CRS, Fuchs AF (2002) The brainstem burst generator for saccadic eye movements: A modern synthesis. Exp Brain Res 142:439-462

Snodderly DM, Kagan I, Gur M (2001) Selective activation of visual cortex neurons by fixational eye movements: implications for neural coding. Visual Neurosci 18:259-277 
Sparks DL (2002) The brainstem control of saccadic eye movements. Nat Rev Neurosci 3:952-964

Sparks DL, Holland R, Guthrie BL (1976) Size and distribution of movement fields in the monkey superior colliculus. Brain Res 113:21-34

Supèr H, van der Togt C, Spekreijse H, Lamme VAF (2004) Correspondence of presaccadic activity in the monkey primary visual cortex with saccadic eye movements. Proc Natl Acad Sci USA 101:3230-3235

Wurtz RH, Goldberg ME (1972) Activity of superior colliculus in behaving monkey. III. Cells discharging before eye movements. J Neurophysiol 35:575-586

Zuber BL, Stark L, Cook G (1965) Microsaccades and the velocity-amplitude relationship for saccadic eye movements. Science 150:1459-1460 


\section{Figure captions}

Fig. 1a-b Illustration of the experimental procedure. (a) Sequences of visual stimulation in the three conditions. (b) Example of a target stimulus display.

Fig. 2a-b Average microsaccade rate evolution over time aligned on target onset (a) and saccade onset (b). (a) Visual-change and memory conditions differ from the visual-static condition in showing second inhibition and enhancement epochs. (b) Rate decreases to near zero prior to response saccades.

Fig. 3 SRTs in visual-static, visual-change, and memory trials conditional on microsaccade occurrence (light bars $=$ no microsaccade; dark bars $=$ microsaccade present) plotted for three different time windows: pre-target (-100-0 ms), target (130-230 ms), and pre-saccade (900-1000 $\mathrm{ms})$. Error bars are SEM. When a microsaccade was detected in the target time window, a decrease in SRTs was found only in the memory condition. An increase was found throughout all conditions after microsaccades in the pre-saccade time window.

Fig. 4 Temporal extension of benefits and costs in saccade latencies after microsaccades. Mean modulations $\triangle \mathrm{SRT}$ in the visual-static, visual-change, and memory condition are presented as a function of time. Shaded areas represent $95 \%$ confidence intervals. Values on the abscissae represent centers of time windows with a width of $\pm 50 \mathrm{~ms}$ used for microsaccade (offset) detection. 
Figure 1
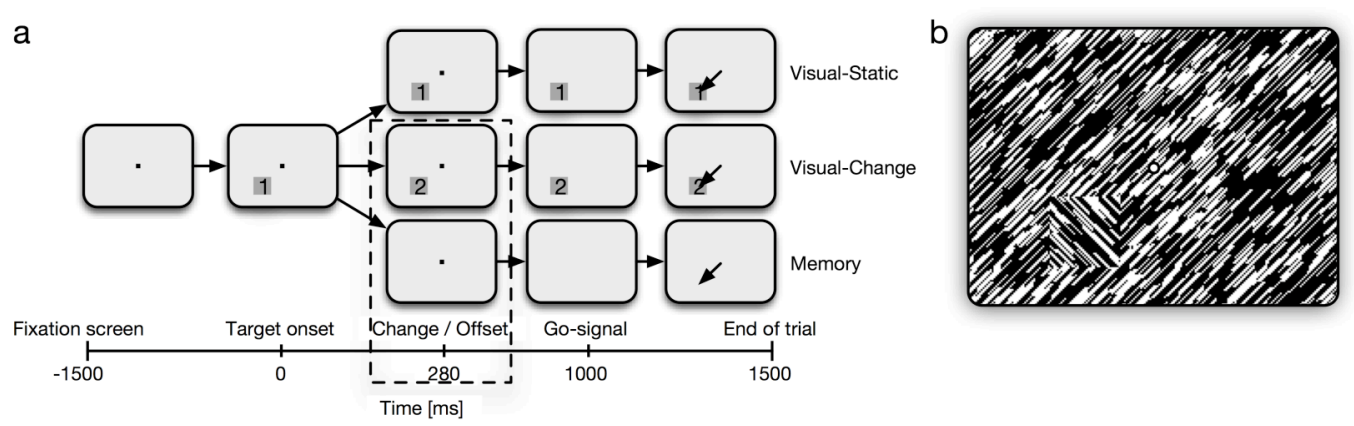

Figure 2

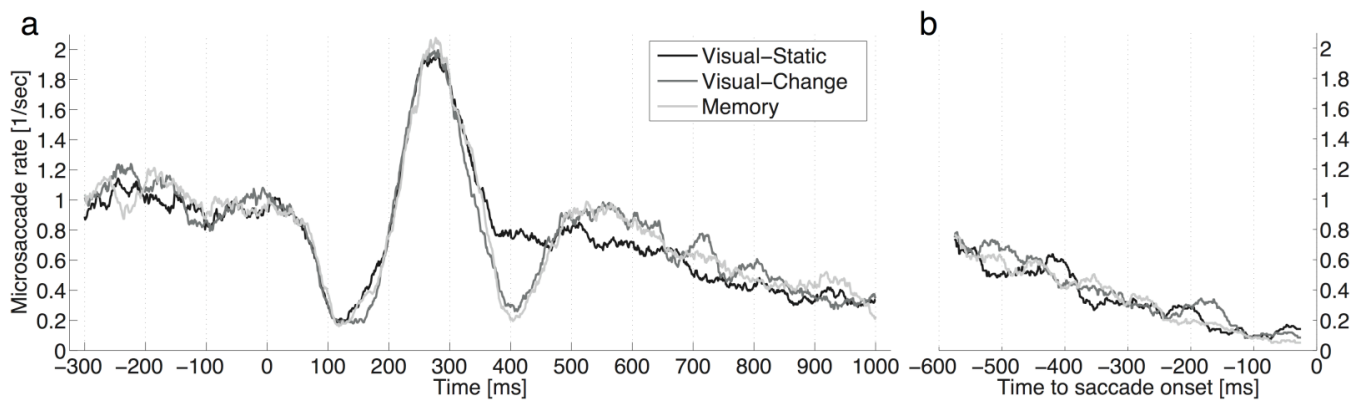

Figure 3

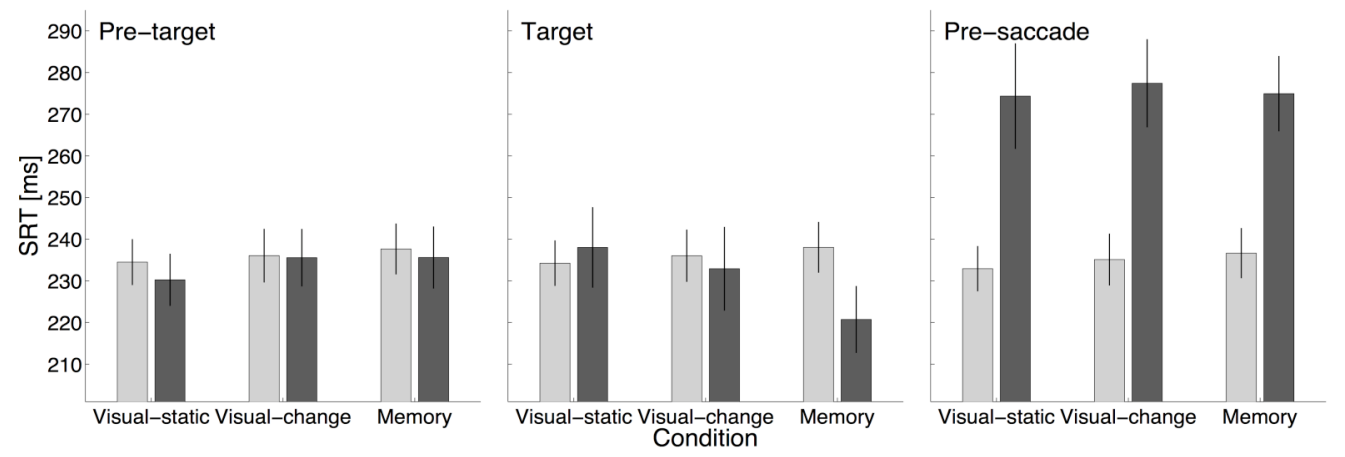


Figure 4

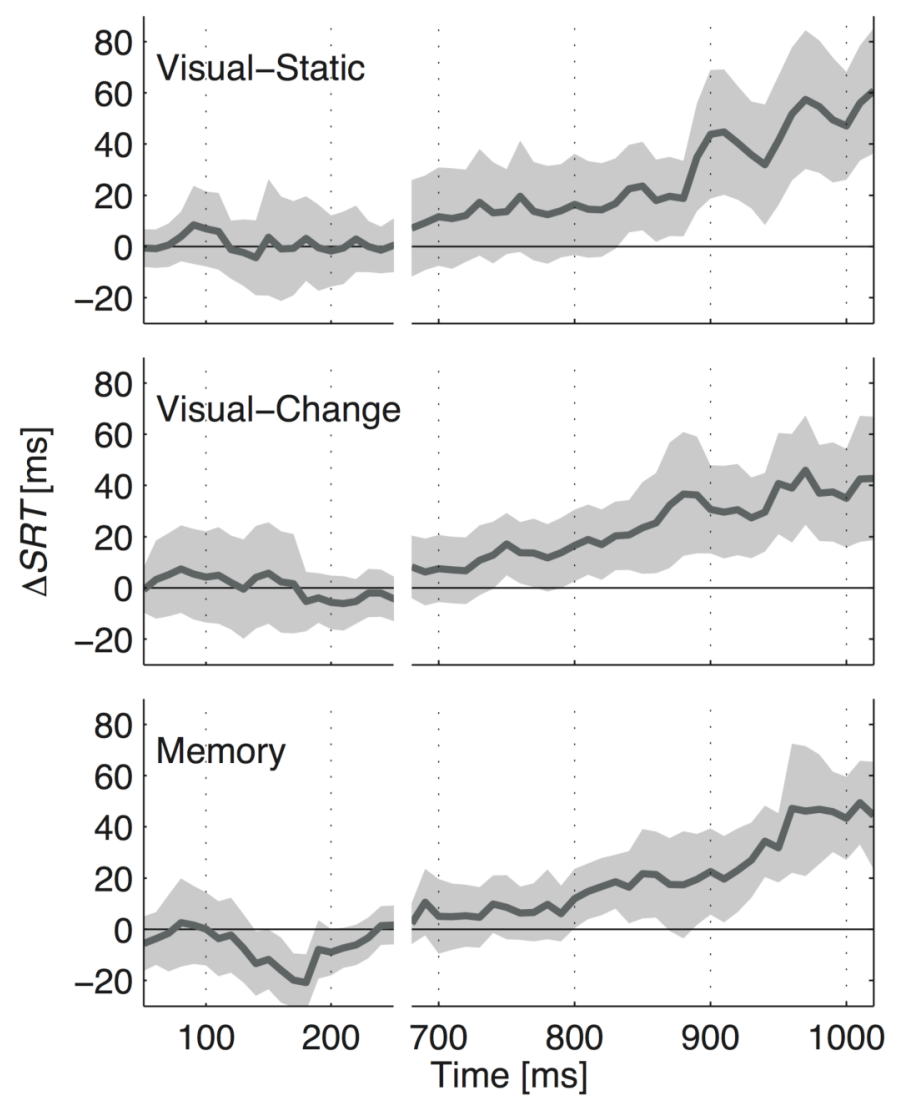

